\title{
A Physics-Based Model for Convolute Current Loss on Z
}

\author{
R. B. Campbell, M. R. Gomez, J.-P. Davis \\ Pulsed Power Sciences Center \\ Sandia National Laboratories, \\ Albuquerque, NM \\ rbcampb@sandia.gov
}

\author{
D. V. Rose, \\ Voss Scientific \\ Albuquerque, NM, United States
}

\begin{abstract}
Sandia's $Z$ machine (D.H. McDaniel, 2002) is a large pulsed-power accelerator used to create extreme conditions of high energy density. Research areas of $Z$ include dynamic properties of materials, radiation and electromagnetic effects on materials and systems, and inertial confinement fusion. In its current embodiment, four magnetically-insulated radial transmission lines (MITLs) are combined at a double post-hole convolute (PHC). Current loss in the convolute can be quite large (20\% of peak load current) for certain pulse shapes and physics loads, thus there is need for a simple physics-based semiempirical model of this current loss in order to accurately predict load-current waveforms using a transmission-line We shall describe the physics contained in the simplified models, show some preliminary results, and discuss how we try to incorporate experimental observations and PIC simulations in our model. We will also describe the inherent uncertainty in the electrode conditions, and how that affects the model results. Based on the model and its integration with BERTHA, recommendations for reducing the current loss will be offered.
\end{abstract}

\section{Keywords—pulsed power system, plasma sources}

\section{INTRODUCTION AND MOTIVATION}

Post-hole convolute geometries are used on the present ZR system (D.H. McDaniel, 2002) in order to add the currents from multiple MITL levels, while maintaining overall low impedance. This particular geometry unfortunately has magnetic nulls at small radius where strong magnetic insulation is absent. These nulls introduce the possibility of parasitic currents reducing the final current to the physics load, thereby reducing performance. Moreover, the results of experiments and particle in cell simulations suggest that additional current paths may exist on the upstream side of the convolute post-holes away from the nulls. It is the purpose of this paper is to describe the issues and models we have considered of cathode plasma production in the post-hole convolute region. The models are of a reduced dimensionality so they can be implemented in a circuit code model such as Bertha (HINSHELWOOD, 1983) for the purpose of prediction and 'post diction' of desired or observed machine current pulse shapes.

\section{Physical CONDition In CONVOLUTE}

\section{A. Geometry}

The four levels of magnetically insulated transmission lines (MITLs) with the post hole convolute and final inner MITL feed is shown in Fig. 1 in cross section. The red structures are the cathodes of the MITL/convolute. The blue structures represent the anodes of the MITLS, electrically connected to the anode posts of the convolute, shown in gold.

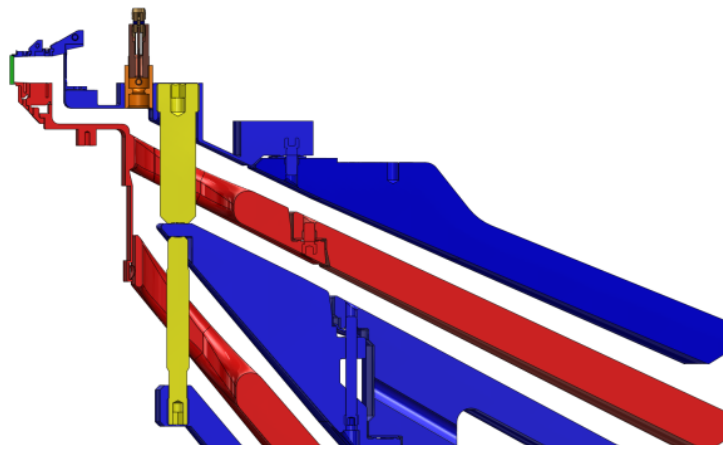

Fig. 1-Section view of the post hole convolute/MITL system, with cut through the anode post (gold). The cathodes are in red, the non-post anodes are in blue. The copper structure is a B-dot monitor used to infer the inner MITL current.

Below is R-theta cross-section of the 12 hole convolute region. Power flows from larger radius inward, magnetic fields increase from larger radius to smaller. The magnetic nulls associated with the holes occur on the upstream side of the hole. The observations of the cathode plasma formation are made in the inner (smaller radius) gap.

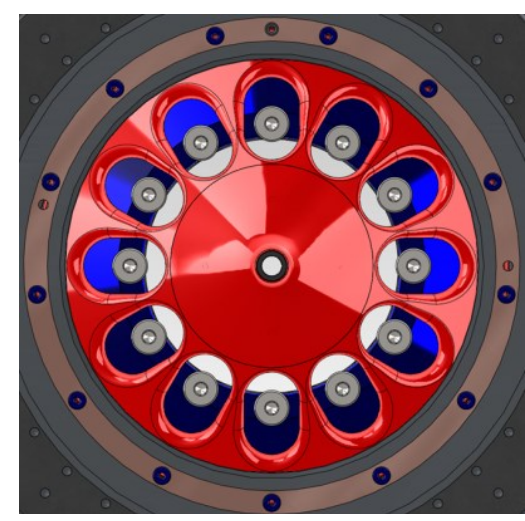

Fig. 2-R-theta cross-sectional view of 12 post-hole convolute. The observations of the cathode plasma formation are made in the inner (smaller radius) gap. 


\section{B. Particle and Plasma Flows}

As the voltage pulse propagates down the MITLs, and the voltage rises in the convolute region, at some point the local electric field exceeds the Child Langmuir space charge limited (SCL) field on various locations on the cathode surface. Exceeding this threshold initiates both an electron current through the magnetic nulls, as well as surface heating which liberates adsorbed contaminants. These contaminants (mostly water vapor and hydrocarbons) ionize and create plasma with an electron temperature of a few electron volts which initially flows through the nulls on the upstream side of the hole, but eventually plasma flows along field lines to the downstream side of the cathode holes and builds to a critical density within a local magnetic well. (D. V. Rose, 2009) Once this critical density is reached, the final current path to the anode posts is a topic of some debate. Simple SCL emission cannot be the mechanism, since the transverse magnetic fields exceed 100T on the downstream side of the convolute holes. There is a belief that particle drifts of energetic electrons in a complicated 3D geometry can be responsible, but underresolved PIC simulations and experiments to date cannot so far give a definitive answer to this question. This open question will have a material effect on the type of model we will propose for the convolute current loss.

\section{EXPERIMENTAL MEASUREMENTS}

In order to develop the best simplified convolute current loss, the available experimental data should be considered and incorporated where possible. In this section we present an abbreviated version of the data that will be discussed at length elsewhere at this conference.

\section{A. Diagnostics}

Measurements of the convolute plasma are taken using a temporally-resolved visible spectroscopy system. The spectral resolution of the system for these measurements was $\sim 2 \AA$ and the temporal resolution was $\sim 2$ ns. The probe used in these experiments is inserted into a vacant inner B-dot hole as shown in Figure 1 (the copper colored structure). Many convolute experiments $(60+)$ were conducted in a "ride-along" mode; no dedicated convolute plasma experiments were performed.

\section{B. Relevant Experiments}

While several classes of experiments have been performed, the two most relevant to the present paper are the measurement of the convolute impedance as a function of time and the apparent convolute A-K gap closure speed. The convolute impedance behavior appears to depend on the duration of time the convolute is under vacuum prior to the shot. This effect will be discussed briefly.

The time history of the convolute impedance is shown in Fig. 3 below. The impedance typically collapses 25-40 nsec after the voltage peak, ending up around 0.02 to $0.5 \mathrm{ohms}$. This data set is comprised of a series of cylindrical liner implosions with nominally identical machine configurations and loads. The wide variation of the final impedance after collapse with nominally the same configuration shows one of the major difficulties in developing a predictive model for convolute current loss. The final state of the current flow indicates that there are hidden variables in the breakdown process that makes model development challenging. From this set of data, we can conclude that the current loss can vary by a factor of several for seemingly identical setups. In the model section for this paper, we will discuss possible sources of and treatment of these hidden variables and random variations in known variables.

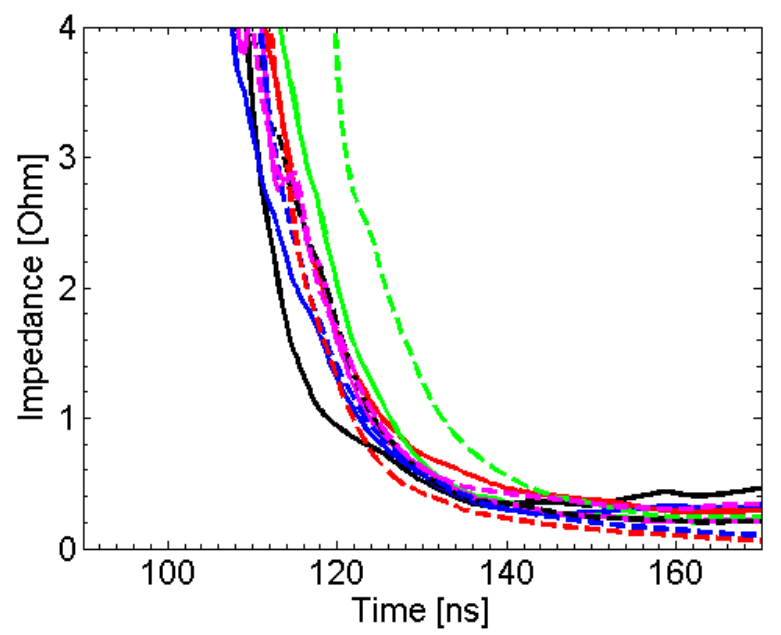

Fig 3-Measured envelope of the convolute impedance time history. The physics load and pulse shapes are quite similar in all of these shots, indicating there is a natural variability in plasma losses.

Measurements from several shots were compared to estimate the plasma closure velocity based on continuum emission turn-on time. Below is typical data for these multi-shot sequences, with the spectrometer pointing at different locations in the convolute A-K gap.

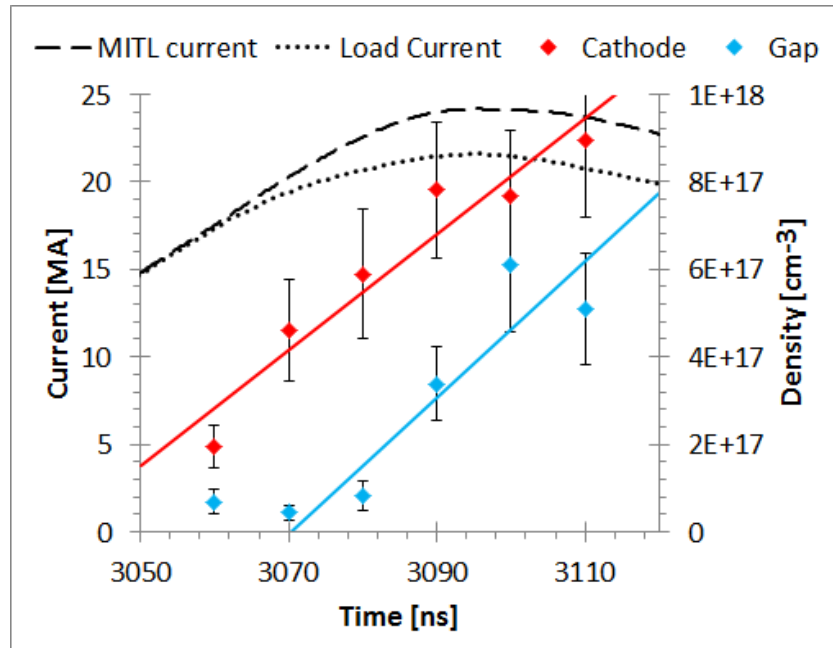

Fig. 4-Measurements of time history of plasma density at the cathode and within the inner gap. 
When one accounts for the fact that the distance between cathode and gap locations for this probe is $4.4 \mathrm{~mm}$, and the time for the front to move between cathode and gap is then we can infer a closure velocity of about $16 \mathrm{~cm} / \mu \mathrm{sec}$. Comparing closure speeds at several times indicates it is relatively constant.

\section{3D PARTICLE IN CELl Simulatons}

In building a simplified model, the more information gathered from various sources, the greater likelihood that the model will represent reality. In section III, we looked at what experimental data can offer us, in this section we seek information from complex, 3D simulations of the convolute and load.

\section{A. The LSP code}

Novel dense plasma modeling techniques, implemented within the 3D PIC code LSP $^{1}$, were used to assess the impact of plasmas desorbed from the cathode "holes" in the $\mathrm{Z}$ convolute (D. V. Rose, 2009). This work suggested that a relatively small plasma desorption rate resulted in net current losses in the convolute of about 1.5 MA, consistent with some $\mathrm{Z}$ load current measurements. We found that some of the plasma desorbed from inner edges of the cathode holes could drift along magnetic field stream lines to collect on the load (inner) side of the anode posts in the gap between these posts and the inner cathode regions of the convolute. This small region of dynamically evolving plasma, located in the otherwise wellinsulated physical gap between the inner cathode hardware and anode posts, resulted in the formation of a small effective cathode-to-anode gap. The small plasma region acted effectively as another cathode surface from which electrons could be drawn across the now small cathode-to-anode gap to the anode posts.

\section{B. Typical ZR convolute simulations}

From the simulations (D. V. Rose, 2009), some plasma penetration into the vertical magnetic field null, extending downward from the upper cathode occurs. However the major source of loss current occurs between the downstream cathode and the upper and lower posts. The plasma penetration downstream results from plasma drifting along magnetic field lines connecting the cathode holes upstream of the posts and travelling along these field lines and collecting downstream of the posts. A small effective AK gap downstream of the posts allows electron current to cross from this plasma region to the anode posts. Since there is strong magnetic insulation on the downstream side, the actual mechanism for the final connection to the post remains a subject of further study. A physical mechanism of energetic particle drifts in the complex $3 \mathrm{D}$ geometry to the posts is a possibility, but definitive conclusions cannot be made due to the gap region being under-resolved. Work is currently underway to assess the

${ }^{1}$ LSP is a software product developed by ATK Mission Research, Albuquerque, NM 87110, with initial support from the Department of Energy SBIR Program. degree of numerical diffusion using smaller domains with denser meshes.

\section{PROPOSED SIMPLIFIED MODEL}

\section{A. Background}

Now that we have examined some experimental data and 3D PIC simulations, some desirable characteristics of the model can be defined.

From the data and knowledge of the process, there are two significant times in the development of the loss current. First, the time at which the initial Child Langmuir space charge limited emissions commences is significant, and could be computed in a simple way by estimating the electric field in the vicinity of the cathode holes. Since from the PIC simulations show a B-field parallel flow of plasma to the inner edge of the cathode holes, there is a finite time for the density to build to a critical level before the final convolute closure occurs. Certainly the plasma sources commence soon after the CL threshold is reached, but it may take until the voltage turns over before the dense plasma can form a virtual cathode in the gaps. (D. V. Rose, 2009)

The distillation of such a complex model into one that can be implemented in a fast running code is a daunting task. In principle, a one-dimensional flux limited fluid model could be developed that describe the desorption of contaminants due to CL emission and anode heating and subsequent along field transport to the inner cathode hole wells, but even this simple model the time per calculation would make BERTHA unusable for the parametric studies currently so important for designing pulse shapes on ZR.

We have considered at least a dozen models for the convolute current loss over the study period to date. Some more complex than others, but a common thread is that they had a space charge limited component, as well as random components related to the surface conditions and effective emission area for plasma generation. Another commonality, and weakness, is that they are based entirely on theoretical considerations. With the final closure mechanism open to current debate, the only prudent model that can be advanced at this point is semiempirical, using knowledge of the general process, combined with data of the onset of convolute impedance collapse.

\section{B. Formulation of the model}

The model will relate the loss current to the voltage across the convolute A-K (post-hole) gap through an effective resistance, $\mathrm{R}_{\text {eff }}$. There are several terms in the general expression, describing different phases of the closure process. First, there is the threshold of space charge limited emission and the accompanying desorption of hydrocarbons and water vapor. For very clean surfaces, minimal desorption is expected, but the pre-shot conditioning currently being used not satisfy the clean surface assumption. The space charge limited flux and the initial plasma flow will be through the magnetic nulls, with an emission area which increases with time, but is limited by the total active area of the nulls. Plasma also collects in wells on the downstream side of the cathode hole. When a 
critical density is reached, determined by the balance between thermal flux and space charge limited flow, the final inner gap closure commences. This timing is on the order of $100 \mathrm{nsec}$ after the null gap closure.

The semi-empirical model takes the following form for a resistive element, $\mathrm{R}_{\mathrm{j}}$ :

$$
\begin{gathered}
R_{j}=C\left\{1-\left[\left(t-t_{0 j}\right) / \tau\right]\right\}^{2} / A_{C j}(t)+D[(t \\
\left.\left.-t_{0 j}\right) / \tau\right] / A_{D j}(t)
\end{gathered}
$$

Where the first term with coefficient ' $\mathrm{C}$ ' is related to Child Langmuir gap closure, and the term with the leading coefficient ' $\mathrm{D}$ ' describes the resistance through the conducting plasma formed in the gaps. The $\tau$ 's are the physical closure times using the experimentally determined gap closure velocity and the inner and outer gap distances. The subscript ' $j$ ' is necessary because we have divided the emission area into regions that have different adsorbed contaminants and electric field profiles for a given mean electric field determined by Bertha. This results in different times, $\mathrm{t}_{0 \mathrm{j}}$, and time dependent areas, $A_{j}$. We typically use five regions for the null gaps and five regions for the downstream gap plasma formation. The number of regions chosen can be varied, and we use the five regions as representative. The profiles of enhanced plasma production are tailored so the resistance shape lies within the envelope of the experimentally determined impedance shown earlier, while at the same time are consistent with the experimental data of the current loss.

\section{RESULTS AND DISCUSSION}

We will first show a pair of shots, Z2333 and Z2450, both used for dynamic materials testing with very similar hardware. Both had similar pre-shot surface preparation, and were pumped down to the $0.02 \mathrm{mTorr}$ level in the center section. The major difference is the initial voltage on the Marx banks used for energy storage. Z2333 was charged to $85 \mathrm{kV}$, and $\mathrm{Z} 2450$ was charged to $53 \mathrm{kV}$. Experiments with higher charge voltage tend to form parasitic plasma in the convolute. To be clear, this is not entirely the case because timings of the MITLs for fixed Marx charge can shape the voltage pulse in ways to either ameliorate or exacerbate this tendency. In some respects, the dynamic material experiments can represent more inherent variability in losses than the radiation and ICF pulse shapes, due to the wide variety of required pulses to provide the required paths of the shocked material load in equation of state space.

We first look at the shot with the lower charge voltage, 2450, shown in fig. 5. There was well below 1 MA of convolute loss for this shot due to the lower Marx charge voltage and the line timing. With parameters in the model consistent with the envelopes of convolute plasma impedance, load current level and most timing aspects are reasonably well represented. One feature that still needs to be modeled is the slower decay after peak current due to inductive effects. This is true for some of the other shots which will be considered later.

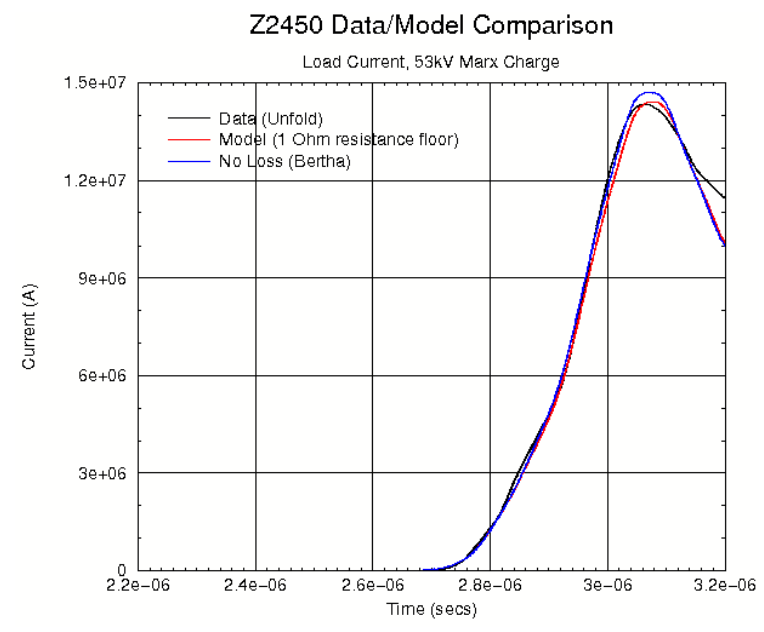

Fig. 5-Load current versus time for a low Marx charge dynamic materials experiment. The black curve is the VISAR unfold (data), and the red curve is the model.

We next consider a shot with a much higher Marx charge, Z2333, in fig. 6. As is clear from the figure, significant losses are present. Compared to the MITL current, there appears to be 3.5 MA of loss current. The resistance envelope in the model captures the load current peak, and nearly the correct timing of the peak. It also appears to have more of the inductive decay as seen in the VISAR unfolded load current than in Z2450.

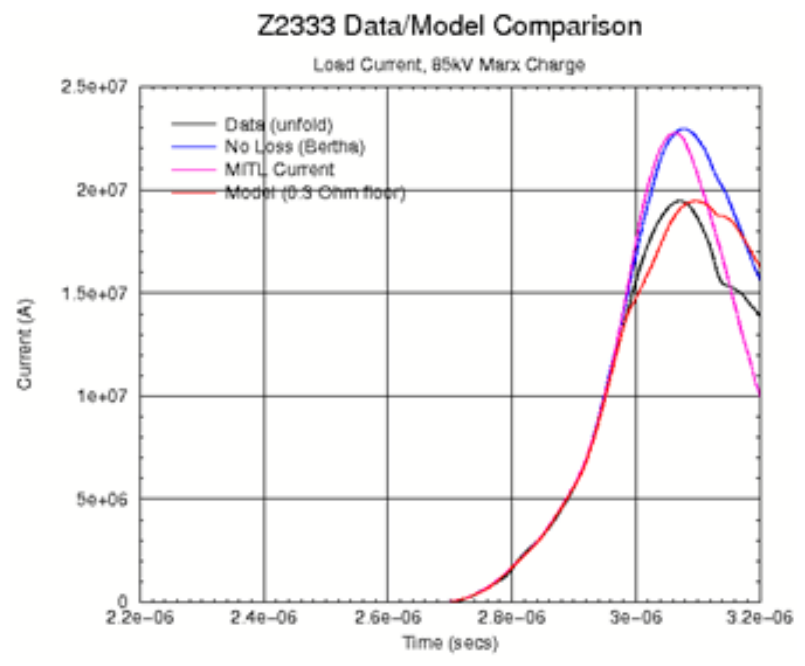

Fig. 6-Load current versus time for a high Marx charge dynamic materials experiment. The black curve is the VISAR unfold (data), and the red curve is the model. More significant losses are evident, based on the magenta and blue curves of MITL and lossless load current respectively.

The final shot comparisons will be between Z2303 and Z2304, shown in fig.7. These two shots are interesting in that one (Z2304) exhibits what would be considered moderate losses, and the other rather high. Although not shown, the lossless 
load current is 19MA, suggesting that Z2303 had 4 MA of loss, whereas Z2304 had only 1.5 MA. The hardware of each shot was very similar, as was the Marx charge, $85 \mathrm{kV}$. The major important difference was that after Z2303 was fielded and had large losses, Z2304 was changed to have a much longer pump down time and lower final center section pressure (0.02 versus $0.002 \mathrm{mTorr})$.

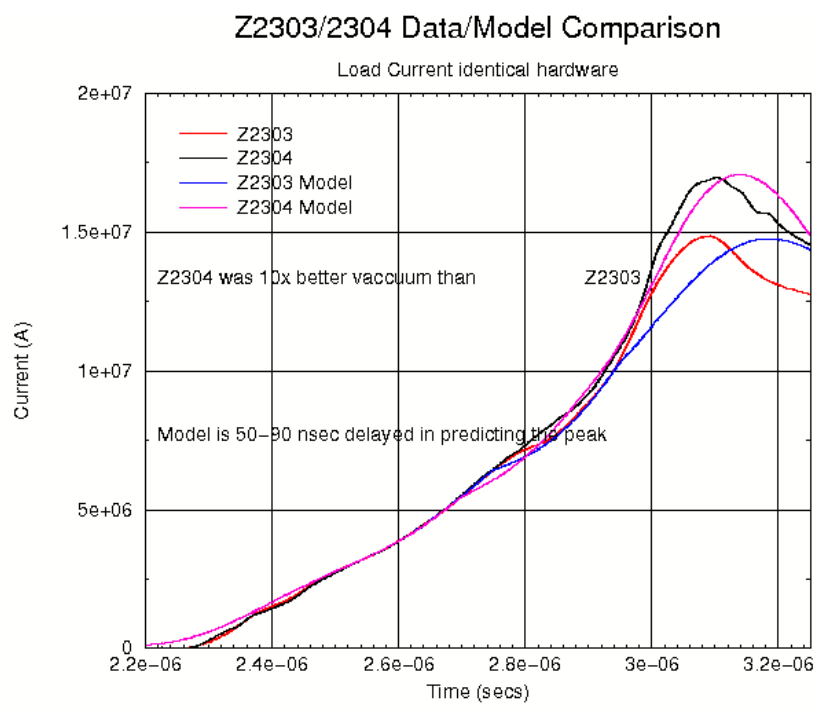

Fig. 7-Load current versus time for two shots with identical loads and switch timings. The difference lies in the degree of surface cleanup (through pumping) was performed. Z2304 had a ambient pressure a factor of 10 below that of Z2303.

This comparison should be a reminder that pre-shot preparation, vacuum, and surface conditions are important aspects of keeping the losses manageable. The model in its present form needs improvement in predicting the timing of peak current, the peaks are delayed 50-90 nsec compared to the data.

While the semi-empirical model can do a fairly reasonable job in describing the current loss in convolutes that is consistent with the resistance profiles measured, it is far from being predictive. This is a natural consequence of the random nature of plasma generation and subsequent gap closure due to dirty surfaces and high electric fields. It may not be ultimately possible to achieve a fully predictive model, particularly one that is compact enough to run efficiently in BERTHA, and suitable for all pulse shapes. One of the possible conclusions of this work is that more emphasis should be placed on the root causes for the current loss, rather than trying to simulate the symptoms by a current loss model.

The standard pre-shot procedure relevant to the convolute behavior for hardware is an alcohol swipe of components. The central section is evacuated to a pressure to a few tens of $\mu$ Torr. The standard finish of the components is a polished stainless steel, although reviewing the shot history there appears to be some hardware that is gold plated, but it was not used routinely.

A dry nitrogen purge could be used to improve surface conditions by reducing water vapor on electrodes. This is common practice in high voltage, high vacuum applications. Another possible pre-shot treatment could be glow discharge cleaning to drive off contaminants, but precious real estate in the vacuum chamber must be set aside for the electrodes of such a system. In addition to the surface treatment protocols, there are some schemes that are used in the accelerator community to increase the Child Langmuir space charge limited threshold. One such approach is that of ion implantation (Sinclair, Dylla, \& al., 2001), where the threshold can be increased by over $40 \%$. Whether this can be replicated in the more hostile $Z$ environment remains to be seen, but might be worthwhile to consider.

There are other alternatives to the MITL/convolute geometry. One that was proposed by VanDevender in 2008, and tested on the Saturn accelerator in 2011 (VanDevender, et al., 2011) is called the Clamshell MITL (CSMITL). The strengths of this design is that the magnetic nulls are located much further out radially, where electric fields are substantially lower. Moreover, the magnetic wells that served to collect plasma on the downstream side of the post-hole convolute are absent in this geometry. Both of these features should reduce parasitic losses upstream of the final feed to the load. The performance of the CSMITL met expectations when it was tested on Saturn when compared to a standard MITL, the load current and power was marginally higher in the CSMITL case. This successful proof of principle may provide impetus for a future full scale test on ZR, however the concept must be proven in a higher power environment.

\section{CONCLUSIONS}

In this brief paper, we have outlined the problem of parasitic current loss on ZR, reviewed the physics as presently understood, as well as described the experimental data and PIC modeling of the relevant processes. We advanced a simple semi-empirical model of convolute resistive loss which was suitable for implementation in the BERTHA circuit code. This model, due to the statistical nature of surface plasma formation, will in all likelihood never be predictive but might be used to bracket the losses for a given pulse shape, using an envelope of experimentally determined convolute impedances. A key finding of this work is that the complexity of the underlying processes suggests working on solutions which address the root causes of the enhanced current loss may be the most fruitful path. While load characteristics (particularly inductance), final desired pulse shape and peak current dictate the electric field distribution and time history in the MITLs and convolute, there can be some actions taken to reduce the current loss under those constraints. 
- In addition to alcohol swiping, purging with dry nitrogen and/or inert gas can clean up surfaces.

- Glow discharge cleaning consistent with the available real estate in the convolute/final feed region could be considered.

- Modifying the stainless steel surface (gold plated or otherwise) with ion implantation to raise the space charge limited electric field threshold.

- $\quad$ Adaptation of the Clamshell MITL design for full ZR operation can move magnetic nulls outboard to lower fields while removing inboard magnetic wells where plasma can form near the final feed.

\section{ACKNOWLEDGMENT}

The authors acknowledge valuable discussions with Dr. Heath L. Hanshaw. Sandia National Laboratories is a multi-program laboratory managed and operated by Sandia Corporation, a wholly owned subsidiary of Lockheed Martin Corporation, for the U.S. Department of Energy's National Nuclear Security Administration under contract DE-AC04 94AL85000.

\section{REFERENCES}

D. V. Rose, e. a. (2009). ZR-CONVOLUTE ANALYSIS AND MODELING: PLASMA EVOLUTION.
Pulsed Power Conference, 2009. PPC '09. IEEE (pp. 1153 - 1158 ). IEEE.

D.H. McDaniel, e. a. (2002). The ZR refurbishment project. Power Modulator Symposium, 2002 and 2002 HighVoltage Workshop. Conference Record of the Twenty-Fifth International, (pp. 252 - 256).

HINSHELWOOD, D. D. (1983). BERTHA -A Versatile Transmission Line and Circuit Code. Washington, D.C.: NAVAL RESEARCH LABORATORY.

Sinclair, C., Dylla, H. F., \& al., e. (2001). Dramatic reduction of DC field emission from large area electrodes by plasma-source ion implantation. Particle Accelerator Conference, 2001. PAC 2001, (pp. 610,612).

VanDevender, J. P., Langston, W., Pasik, M., Coats, R., Pointon, T., Seidel, D., et al. (2011). New selfmagnetically insulated connection of multi-level accelerators to a common load. Pulsed Power Conference (PPC), 2011 IEEE (pp. 1003,1008). IEEE Press. 